Georgian Mathematical Journal

Volume 8 (2001), Number 2, 307-318

\title{
ENTROPY NUMBERS OF DIAGONAL OPERATORS OF LOGARITHMIC TYPE
}

\author{
THOMAS KÜHN \\ Dedicated to Professor Nicholas Vakhania \\ on his 70th birthday
}

\begin{abstract}
We determine the asymptotic behaviour (as $k \rightarrow \infty$, up to multiplicative constants not depending on $\mathrm{k})$ of the entropy numbers $e_{k}\left(D_{\sigma}\right.$ : $\left.l_{p} \rightarrow l_{q}\right), 1 \leq p \leq q \leq \infty$, of diagonal operators generated by logarithmically decreasing sequences $\sigma=\left(\sigma_{n}\right)$. This complements earlier results by Carl [2] who investigated the case of power-like decay of the diagonal.
\end{abstract}

2000 Mathematics Subject Classification: 47B06, 46B45, 46B70, $28 \mathrm{C} 20$.

Key words and phrases: Entropy numbers, sequence spaces, complex interpolation, Gaussian processes.

\section{INTRODUCTION}

In the last few years there has appeared quite a number of research papers (see [17], [18], [7] and [8]) and several monographs (see [9] and [25]) on compact embeddings of function spaces in so-called limiting situations, where the spaces have the same 'differential dimension'. The 'degree' of compactness of a (bounded linear) operator can be measured by the rate of decay of its entropy numbers. Standard tools in the treatment of such embedding problems are discretization techniques, which allow to reduce an infinite dimensional problem to a finite dimensional one, or which reduce function space embeddings to embeddings of weighted vector-valued sequence spaces. This approach requires sharp estimates for the entropy numbers of the latter embeddings. Equivalently, by shifting the weights from spaces to operators, one can consider diagonal operators in sequence spaces without weights.

For classical non-limiting embeddings these weights are of power-like decay. In the above-mentioned limiting situations, however, the resulting weights decrease only logarithmically. This was one of the motivations for the present work.

A further motivation came from the paper by Carl [2] who determined the entropy behaviour of diagonal operators $D_{\sigma}: l_{p} \rightarrow l_{q}, 1 \leq p, q \leq \infty$, in the case where the diagonal $\sigma$ belongs to some Lorentz space $l_{r, t}$. Typically, for 
$t=\infty$, this means $\sigma_{n} \preceq n^{-\alpha}$ with $\alpha=1 / r$. We give in this paper sharp entropy estimates for diagonal operators $D_{\sigma}$ in the following two cases:

(P) $\sigma_{n} \sim n^{-\alpha}(1+\log n)^{\delta}, 1 \leq p, q \leq \infty, \alpha>\max (1 / q-1 / p, 0), \delta \in \mathbb{R}$;

(L) $\sigma_{n} \sim(1+\log n)^{-\delta}, 1 \leq p \leq q \leq \infty, \delta>0$.

The first case $(\mathrm{P})$, of power-like decay of the diagonal, is a variant of Carl's result [2], and the proof also relies on the same, by now standard, technique of splitting the operator $D_{\sigma}$ in dyadic parts. The logarithmic case (L), however, extends the results of [2] to the limiting situation $r=\infty$ or, equivalently, $\alpha=0$. Here the dyadic splitting does not give satisfactory estimates and so a new technique is needed.

Our new approach (recently introduced in two joint papers of the author with Cobos [5] and Schonbek [15]) is essentially based on a close relation between Gaussian processes (resp. Gaussian measures) and entropy. More precisely, we use Sudakov's famous minoration principle - in the form of an inequality between entropy numbers and the $l$-norm of an operator - in combination with certain interpolation techniques.

The organization of the paper is as follows. In Section 2 we fix the notation, and give a few preliminary results which will be needed in the sequel. In Section 3 the main results are formulated, while Section 4 contains their proofs.

\section{Notation and Preliminary Results}

a. Entropy numbers and ideals. The $k^{\text {th }}$ (dyadic) entropy number of a bounded linear operator $T: X \rightarrow Y$ from a Banach space $X$ into another Banach space $Y$ is defined as

$$
e_{k}(T)=\inf \left\{\varepsilon>0: T\left(B_{X}\right) \text { can be covered by } 2^{k-1} \text { balls of radius } \varepsilon \text { in } Y\right\},
$$

where $B_{X}$ denotes the closed unit ball in $X$. Entropy numbers are closely related to Kolmogorov's concept of metric entropy which dates back to the 1930's and which since then has been successfully applied in various fields of mathematics. For basic properties of entropy numbers and applications to eigenvalue and compactness problems we refer to the monographs by Pietsch [21], König [12], Carl and Stephani [4], Edmunds and Triebel [9], and Triebel [25], and the references given therein; for recent applications see also Edmunds and Haroske $[7,8]$, and Leopold $[17,18]$.

We use the common notation $l_{p}^{n}$ for the space $\mathbb{R}^{n}$ (or $\mathbb{C}^{n}$ ) equipped with the quasi-norm (which is even a norm for $p \geq 1$ )

$$
\|x\|_{p}= \begin{cases}\left(\sum_{k=1}^{n}\left|x_{k}\right|^{p}\right)^{1 / p} & \text { for } \quad 0<p<\infty \\ \sup _{1 \leq k \leq n}\left|x_{k}\right| & \text { for } \quad p=\infty .\end{cases}
$$

Given two sequences $\left(a_{k}\right)_{k=1}^{\infty}$ and $\left(b_{k}\right)_{k=1}^{\infty}$ of positive real numbers, we shall write $a_{k} \preceq b_{k}$ if there is a constant $c>0$ such that the inequality $a_{k} \leq c \cdot b_{k}$ holds for all $k \in \mathbb{N}$, and $a_{k} \sim b_{k}$ if both $a_{k} \preceq b_{k}$ and $b_{k} \preceq a_{k}$ are fulfilled. Logarithms are always taken to base $2, \log =\log _{2}$. 
The following basic result is due to Schütt [22] who determined the exact asymptotic behaviour of entropy numbers of the formal identity between finite dimensional $l_{p}$-spaces.

Lemma 1. Let $1 \leq p \leq q \leq \infty$, then (in the real case)

$$
e_{k}\left(i d: l_{p}^{n} \rightarrow l_{q}^{n}\right) \sim\left\{\begin{array}{cl}
1 & \text { if } 1 \leq k \leq \log n \\
\left(\frac{\log \left(\frac{n}{k}+1\right)}{k}\right)^{1 / p-1 / q} & \text { if } \log n \leq k \leq n \\
2^{-\frac{k-1}{n} n^{1 / q-1 / p}} & \text { if } k \geq n .
\end{array}\right.
$$

Remark 1. Formula (1) remains valid for complex spaces if on the right-hand side $n$ is replaced by $2 n$. Roughly speaking, this follows from volume arguments, regarding the $n$-dimensional complex spaces $l_{p}^{n}$ as $2 n$-dimensional real spaces.

Remark 2. A consequence of the special case $k=n$ and elementary properties of entropy numbers is the relation

$$
e_{n}\left(i d: l_{p}^{n} \rightarrow l_{q}^{n}\right) \sim n^{1 / q-1 / p} \text { for all } 1 \leq p, q \leq \infty .
$$

The asymptotic relations (1) are fundamental for numerous applications, as explained in the Introduction. In passing, we mention that (1) has recently been extended to the quasi-Banach case $0<p \leq q \leq \infty$, see $[9,25,14]$. This was again motivated by applications, mainly by the increasing interest during the last decade in quasi-Banach function spaces, for instance in (both classical and generalized) Besov, Sobolev, and Lipschitz spaces.

Another very useful result is due to Gordon, König and Schütt [11, Proposition 1.7.].

Lemma 2. Let $X$ be a real Banach space with 1-unconditional basis $\left\{x_{j}\right\}_{j=1}^{\infty}$ (for example a symmetric Banach sequence space, or any of the spaces $l_{p}$ with $1 \leq p<\infty)$, and let $D_{\sigma}: X \rightarrow X$ be the diagonal operator induced by $x_{j} \mapsto$ $\sigma_{j} x_{j}$, where $\sigma_{1} \geq \sigma_{2} \geq \cdots \geq 0$. Then

$$
\sup _{n \in \mathbb{N}} 2^{-k / n}\left(\sigma_{1} \cdots \sigma_{n}\right)^{1 / n} \leq e_{k+1}\left(D_{\sigma}\right) \leq 6 \cdot \sup _{n \in \mathbb{N}} 2^{-k / n}\left(\sigma_{1} \cdots \sigma_{n}\right)^{1 / n}
$$

for all $k \in \mathbb{N}$.

Remark 3. Inequality (3) remains valid for complex spaces if the factor $2^{-k / n}$ is replaced by $2^{-k / 2 n}$.

As usual, we denote by $l_{p, q}, 0<p<\infty, 0<q \leq \infty$, the Lorentz sequence space consisting of all (real or complex) null sequences $\left(x_{k}\right)_{k=1}^{\infty}$ such that the quasi-norm

$$
\|x\|_{p, q}:=\left\{\begin{array}{lll}
\left(\sum_{k=1}^{\infty} k^{q / p-1}\left|x_{k}^{*}\right|^{q}\right)^{1 / q} & \text { if } & 0<q<\infty \\
\sup _{1 \leq k<\infty} k^{1 / p}\left|x_{k}^{*}\right| & \text { if } & q=\infty
\end{array}\right.
$$


is finite. Here the non-increasing rearrangement $\left(x_{k}^{*}\right)$ of the sequence $\left(x_{k}\right)$ is defined by $x_{k}^{*}=x_{\pi(k)}$, where $\pi: \mathbb{N} \rightarrow \mathbb{N}$ is a permutation with $\left|x_{\pi(1)}\right| \geq\left|x_{\pi(2)}\right| \geq$ $\cdots \geq 0$. All Lorentz spaces are quasi-Banach spaces, and for $1<p<\infty$, $1 \leq q \leq \infty$ and $p=q=1$ the quasi-norms $\|\cdot\|_{p, q}$ are even equivalent to norms. In particular we have $l_{p, p}=l_{p}$ with equality of (quasi)-norms.

Moreover, for the same range of indices $p, q$, we shall use the entropy ideals $\mathcal{L}_{p, q}^{(e)}$, formed by all bounded linear operators $T$ between Banach spaces such that the quasi-norm

$$
L_{p, q}^{(e)}(T):=\left\|\left(e_{n}(T)\right)\right\|_{p, q}
$$

is finite. The classes $\left(\mathcal{L}_{p, q}^{(e)}, L_{p, q}^{(e)}\right)$ are quasi-normed operator ideals in the sense of Pietsch [21, Definition 6.1.1].

Given any sequence $\sigma=\left(\sigma_{k}\right)_{k=1}^{\infty}$, the diagonal operator $D_{\sigma}$ is defined by

$$
D_{\sigma}\left(x_{k}\right):=\left(\sigma_{k} x_{k}\right)
$$

Throughout the paper we shall assume that $\sigma_{1} \geq \sigma_{2} \geq \cdots \geq 0$. We state now the result of Carl [2] on the entropy numbers of diagonal operators.

Lemma 3. Let $1 \leq p, q \leq \infty, 0<r, s<\infty$ and $0<t \leq \infty$ such that $1 / s:=1 / r+1 / p-1 / q>0$. Then one has the equivalence

$$
\sigma=\left(\sigma_{k}\right) \in l_{r, t} \Longleftrightarrow D_{\sigma} \in \mathcal{L}_{s, t}^{(e)}\left(l_{p}, l_{q}\right)
$$

Remark 4 . In the proof the following estimate, for $s>0$ with $1 / s>1 / p-$ $1 / q>0$

$$
L_{s, \infty}^{(e)}\left(i d: l_{p}^{n} \rightarrow l_{q}^{n}\right) \sim n^{1 / s+1 / q-1 / p}
$$

was used, which in turn follows from (1) and the elementary properties of entropy numbers.

b. Gaussian measures and entropy. In this subsection all spaces are assumed to be real. The $l$-norm (introduced by Linde and Pietsch [19] under the name of $\pi_{\gamma}$-norm) of an operator $T$ from a separable Hilbert space $H$ into a Banach space $X$ is defined as

$$
l(T)=\sup _{1 \leq i \leq n}\left(\mathbb{E}\left\|\sum_{i=1}^{n} T e_{i} g_{i}\right\|^{2}\right)^{1 / 2},
$$

where $\left(e_{i}\right)$ is an arbitrary orthonormal basis in $H$ and $\left(g_{i}\right)$ a sequence of i.i.d. standard normal random variables. The $l$-norm is closely related to Gaussian measures on Banach spaces (see, e.g., the monograph by Ledoux and Talagrand [16]) and is an important tool in Banach space geometry (see the book by Pisier $[20]$ ). We only need two basic and well-known facts, the first one can be found, e.g., in [19, Theorem 9]. 
Lemma 4. There are constants $0<c<C<\infty$ such that the estimate

$$
c \cdot \sup _{n \in \mathbb{N}} \sigma_{n} \sqrt{1+\log n} \leq l\left(D_{\sigma}\right) \leq C \cdot \sup _{n \in \mathbb{N}} \sigma_{n} \sqrt{1+\log n}
$$

holds for every diagonal operator $D_{\sigma}: l_{2} \rightarrow l_{\infty}$, with $\sigma_{1} \geq \sigma_{2} \geq \cdots \geq 0$.

The next result is known as Sudakov's minoration principle, see [23] for the original formulation, [16, Theorem 3.18] for a variant in the language of Gaussian processes, or [13] for the version given below.

Lemma 5. There is a constant $c>0$ such that the inequality

$$
\sup _{k \in \mathbb{N}} k^{1 / 2} e_{k}\left(T^{*}\right) \leq c \cdot l(T)
$$

holds for all operators $T$ from a Hilbert space $H$ into a Banach space $X$. (Here $T^{*}$ denotes the dual of $T$.)

The two preceding lemmas immediately imply

Lemma 6. There is a constant $c>0$ such that for the diagonal operator $D_{\sigma}: l_{1} \rightarrow l_{2}$, generated by $\sigma_{k}=(1+\log k)^{-1 / 2}$, the inequality

$$
e_{k}\left(D_{\sigma}\right) \leq c \cdot k^{-1 / 2}
$$

holds for all $k \in \mathbb{N}$.

Remark 5. By standard considerations one can easily show that this result remains true for complex spaces as well.

c. Complex interpolation and entropy. In this subsection we shall consider only complex Banach spaces since the complex interpolation method does not make sense for real spaces. Our general references to interpolation theory, in particular to the complex method, are the monographs by Bergh and Löfström [1], and Triebel [26]; we shall use the same notation and terminology, and state some known facts for later applications.

First we fix some notation. If $0<\theta<1$ and $\left(A_{0}, A_{1}\right)$ is an interpolation couple (i.e., a pair of Banach spaces which both embed linearly and continously into a common Hausdorff vector space), then $\left[A_{0}, A_{1}\right]_{\theta}$ denotes as usual the complex interpolation space of the couple.

If $A$ is a Banach space and $\lambda>0$ a positive real number, then $\lambda A$ means the same Banach space $A$, but equipped with the norm

$$
\|x\|_{\lambda A}:=\lambda \cdot\|x\|_{A} \text {. }
$$

Given $1 \leq p \leq \infty$ and Banach spaces $A_{n}, n \in \mathbb{N}$, we denote by $l_{p}\left(A_{n}\right)$ the set of all sequences $x=\left(x_{n}\right)_{n}^{\infty}$ such that $x_{n} \in A_{n}$ for all $n \in \mathbb{N}$ and

$$
\left(\left\|x_{n}\right\|_{A_{n}}\right)_{n=1}^{\infty} \in l_{p} \text {. }
$$

The vector-valued spaces $l_{p}\left(A_{n}\right)$ are Banach spaces with respect to the norm

$$
\|x\|:=\left\|\left(\left\|x_{n}\right\|_{A_{n}}\right)_{n=1}^{\infty}\right\|_{p} .
$$


In the particular case $A_{n}=w_{n} \mathbb{C}$, with $w_{n}>0$ for all $n \in \mathbb{N}$, we obtain weighted $l_{p}$-spaces, simply denoted by $l_{p}(w)$, where $w=\left(w_{n}\right)_{n=1}^{\infty}$ is the weight sequence. The following known interpolation formula for weighted $l_{p}$-spaces will be needed later.

Lemma 7. Let $1 \leq p_{0}, p_{1} \leq \infty$, but not $p_{0}=p_{1}=\infty$, let $0<\theta<1$, and let $w_{0}$ and $w_{1}$ be arbitrary weight sequences. Then one has with equality of norms

$$
\left[l_{p_{0}}\left(w_{0}\right), l_{p_{1}}\left(w_{1}\right)\right]_{\theta}=l_{p}\left(w_{0}^{1-\theta} w_{1}^{\theta}\right), \text { where } \frac{1}{p}=\frac{1-\theta}{p_{0}}+\frac{\theta}{p_{1}} .
$$

Proof. From the very definition of the complex method it follows that for every Banach space $A$ and arbitrary real constants $\lambda_{0}, \lambda_{1}>0$ one has equality of norms

$$
\left[\lambda_{0} A, \lambda_{1} A\right]_{\theta}=\lambda A, \text { where } \lambda=\lambda_{0}{ }^{1-\theta} \lambda_{1}{ }^{\theta} .
$$

Moreover, given an arbitrary sequence of Banach couples $\left(A_{n}, B_{n}\right), n \in \mathbb{N}$, the following interpolation formula for vector-valued $l_{p}$-spaces

$$
\left[l_{p_{0}}\left(A_{n}\right), l_{p_{1}}\left(B_{n}\right)\right]_{\theta}=l_{p}\left(\left[A_{n}, B_{n}\right]_{\theta}\right), \text { where } \frac{1}{p}=\frac{1-\theta}{p_{0}}+\frac{\theta}{p_{1}},
$$

holds, also with equality of norms, see, e.g., [26, Theorem 1.18.1. and Remarks 1 and 2, p. 122]. From (8) combined with (7), assertion (6) follows.

Finally let us recall the nice behaviour of entropy numbers under interpolation, specified to the complex method, which is sufficient for our present purposes. The general result can be found in [21, Theorems 12.1.11 and 12.1.12]. Here $\mathcal{L}(X, Y)$ stands for the class of all bounded linear operators from $\mathrm{X}$ into $\mathrm{Y}$.

Lemma 8. Let $A$ and $B$ be Banach spaces, $\left(A_{0}, A_{1}\right)$ and $\left(B_{0}, B_{1}\right)$ interpolation couples, $0<\theta<1$ and $m, n \in \mathbb{N}$. Then the following holds:

(i) If $T \in \mathcal{L}\left(A_{0}+A_{1}, B\right)$, then

$$
e_{m+n-1}\left(T:\left[A_{0}, A_{1}\right]_{\theta} \rightarrow B\right) \leq 2 e_{m}\left(T: A_{0} \rightarrow B\right)^{1-\theta} e_{n}\left(T: A_{1} \rightarrow B\right)^{\theta} .
$$

(ii) If $T \in \mathcal{L}\left(A, B_{0} \cap B_{1}\right)$, then

$$
e_{m+n-1}\left(T: A \rightarrow\left[B_{0}, B_{1}\right]_{\theta}\right) \leq 2 e_{m}\left(T: A \rightarrow B_{0}\right)^{1-\theta} e_{n}\left(T: A \rightarrow B_{1}\right)^{\theta} .
$$

\section{Main Results}

Now we state our main results on the entropy numbers of diagonal operators. The first two theorems deal with the case, where the diagonal decreases essentially as a power.

Theorem 1. Let $1 \leq p, q \leq \infty, \alpha>\max (1 / q-1 / p, 0)$ and $\delta \in \mathbb{R}$. Then one has the implication

$$
\sigma_{n} \sim n^{-\alpha}(1+\log n)^{\delta} \Longrightarrow e_{n}\left(D_{\sigma}: l_{p} \rightarrow l_{q}\right) \sim n^{1 / q-1 / p-\alpha}(1+\log n)^{\delta} .
$$


Theorem 2. Under the assumptions of Theorem 1 one has the equivalence

$$
\sigma_{n} \preceq n^{-\alpha}(1+\log n)^{\delta} \Longleftrightarrow e_{n}\left(D_{\sigma}: l_{p} \rightarrow l_{q}\right) \preceq n^{1 / q-1 / p-\alpha}(1+\log n)^{\delta} .
$$

It seems appropriate to give here some brief comments on the relation of our results (Theorems 1 and 2) and Carl's result [2] (stated in Lemma 3). The main difference lies in the way of measuring the rate of decay of a positive decreasing sequence $\sigma=\left(\sigma_{n}\right)$ of real numbers. While in [2] membership to a Lorentz space $l_{r, t}$ was used, we prefer to work instead with the more direct conditions $\sigma_{n} \preceq n^{-\alpha}(1+\log n)^{\delta}$. Both ways provide a two-parameter scale of conditions with the 'main' parameter $(r$, resp. $\alpha$ ) and the 'fine' parameter $(t, \operatorname{resp} . \delta)$. Although quite close to each other, the two scales are different, their only 'intersection point' being the case $\left(\sigma_{n}\right) \in l_{r, \infty} \Longleftrightarrow \sigma_{n} \preceq n^{-1 / r}$. In a certain sense our scale is more sensible. For example, the Lorentz scale cannot distuingish between the conditions $\sigma_{n} \preceq n^{-\alpha}(1+\log n)^{\delta}$, where $\alpha>0$ is fixed and $\delta>0$ is arbitrary.

Now we turn to the case of logarithmic decay of the diagonal $\sigma$, which corresponds to the limiting situation $\alpha=0$ in the preceding theorem. Of course, in order to obtain a bounded operator from $l_{p}$ into $l_{q}$, we have to require $p \leq q$. For $p=q$ the entropy behaviour of $D_{\sigma}$ is known, see Remark 6 below.

Theorem 3. Let $1 \leq p<q \leq \infty$ and $\delta>0$, and set $\delta^{*}:=1 / p-1 / q$. Then the condition $\sigma_{n} \sim(1+\log n)^{-\delta}$ implies

$$
e_{n}\left(D_{\sigma}: l_{p} \rightarrow l_{q}\right) \sim\left\{\begin{array}{cl}
n^{1 / q-1 / p}(1+\log n)^{1 / p-1 / q-\delta} & \text { if } \delta \geq \delta^{*} \\
n^{-\delta} & \text { if } \delta \leq \delta^{*}
\end{array}\right.
$$

Theorem 4. Under the assumptions of Theorem 3 the condition $\sigma_{n} \preceq(1+$ $\log n)^{-\delta}$ is equivalent to

$$
e_{n}\left(D_{\sigma}: l_{p} \rightarrow l_{q}\right) \preceq\left\{\begin{array}{cl}
n^{1 / q-1 / p}(1+\log n)^{1 / p-1 / q-\delta} & \text { if } \delta \geq \delta^{*} \\
n^{-\delta} & \text { if } \delta \leq \delta^{*} .
\end{array}\right.
$$

Remark 6 . Note that in the case $p=q, \delta>0, \sigma_{n} \sim(1+\log n)^{-\delta}$ the result of Gordon, König and Schütt [11], stated here as Lemma 2, implies

$$
e_{n}\left(D_{\sigma}: l_{p} \rightarrow l_{p}\right) \sim(1+\log n)^{-\delta},
$$

which in contrast to Theorem 2 means that we have only logarithmic decay of the entropy numbers. This shows that the embeddding $l_{p} \hookrightarrow l_{q}$ contributes essentially to the power-like decay of the entropy numbers.

Another new feature of the entropy behaviour of diagonal operators with logarithmic diagonals, compared to power-like diagonals, is the existence of the 'critical' value $\delta^{*}$, where the asymptotic entropy behaviour changes drastically. For $\delta \geq \delta^{*}$ the entropy numbers keep almost stable, up to logarithmic factors. A similar phenomenon has occured recently in the study of entropy numbers 
of convex hulls, see the papers by Carl, Kyrezi and Pajor [3], Gao [10], and Creutzig and Steinwart [6].

\section{Proofs}

Now we give the proofs of our main results.

Proof of Theorem 1. Upper estimate:

For all integers $n \geq 0$ consider the dyadic parts $D_{n}$ of the operator $D_{\sigma}$, defined by

$$
D_{n} x=\left(0, \ldots, 0, \sigma_{2^{n}} x_{2^{n}}, \ldots, \sigma_{2^{n+1}-1} x_{2^{n+1}-1}, 0, \ldots\right) .
$$

Then, clearly,

$$
D_{\sigma}=\sum_{n=0}^{\infty} D_{n}
$$

Choose $s>0$ with $1 / s>\alpha+1 / p-1 / q$, and observe that the quasi-norm $L_{s, \infty}^{(e)}$ is equivalent to an $r$-norm, for appropriate $0<r<1$. Now, for any fixed $N \in \mathbb{N}$, it follows from (5) and the multiplicativity of entropy numbers that

$$
\begin{aligned}
& L_{s, \infty}^{(e)}\left(\sum_{n=0}^{N} D_{n}\right)^{r} \leq c \sum_{n=0}^{N} L_{s, \infty}^{(e)}\left(D_{n}\right)^{r} \leq c \sum_{n=0}^{N} \sigma_{2^{n}}^{r} L_{s, \infty}^{(e)}\left(i d: l_{p}^{2^{n}} \rightarrow l_{q}^{2^{n}}\right)^{r} \\
& \sim \sum_{n=0}^{N}\left(2^{-\alpha n} n^{\delta} \cdot 2^{(1 / s+1 / q-1 / p) n}\right)^{r} \sim\left(N^{\delta} 2^{(1 / s+1 / q-1 / p-\alpha) N}\right)^{r} .
\end{aligned}
$$

This implies in particular

$$
e_{2^{N}}\left(\sum_{n=0}^{N} D_{n}\right) \preceq N^{\delta} 2^{(1 / q-1 / p-\alpha) N} .
$$

Now we estimate the entropy numbers of the tail of the sum (9). To this end, we choose now $s>0$ with $1 / p-1 / q<1 / s<\alpha+1 / p-1 / q$. Again, we use the fact that the quasi-norm $L_{s, \infty}^{(e)}$ is equivalent to an $r$-norm, and so we can estimate it as above (that we have now different $r$ and $s$ does not matter, this influences only the constants):

$$
\begin{aligned}
& L_{s, \infty}^{(e)}\left(\sum_{n=N+1}^{\infty} D_{n}\right)^{r} \leq c \sum_{n=N+1}^{\infty} L_{s, \infty}^{(e)}\left(D_{n}\right)^{r} \\
& \leq c \sum_{n=N+1}^{\infty} \sigma_{2^{n}}^{r} L_{s, \infty}^{(e)}\left(i d: l_{p}^{2^{n}} \rightarrow l_{q}^{2^{n}}\right)^{r} \\
& \sim \sum_{n=N+1}^{\infty}\left(2^{-\alpha n} n^{\delta} \cdot 2^{(1 / s+1 / q-1 / p) n}\right)^{r} \sim\left(N^{\delta} 2^{(1 / s+1 / q-1 / p-\alpha) N}\right)^{r} .
\end{aligned}
$$

It follows that

$$
e_{2^{N}}\left(\sum_{n=N+1}^{\infty} D_{n}\right) \preceq N^{\delta} 2^{(1 / q-1 / p-\alpha) N}
$$


and by the additivity of entropy numbers we conclude from (10) and(11)

$$
e_{2 \cdot 2^{N}}\left(D_{\sigma}\right) \leq e_{2^{N}}\left(\sum_{n=0}^{N} D_{n}\right)+e_{2^{N}}\left(\sum_{n=N+1}^{\infty} D_{n}\right) \preceq N^{\delta} 2^{(1 / q-1 / p-\alpha) N},
$$

which proves the upper estimate since $N \in \mathbb{N}$ was arbitrary.

Lower estimate. For arbitrary $n \in \mathbb{N}$, let $R_{n}$ denote the restriction of $D_{\sigma}$ to the coordinates $1, \ldots, n$. Then the multiplicativity of entropy numbers and (1) imply the desired relation

$$
e_{n}\left(D_{\sigma}\right) \geq e_{n}\left(R_{n}\right) \geq \sigma_{n} \cdot e_{n}\left(i d: l_{p}^{n} \rightarrow l_{q}^{n}\right) \sim n^{1 / q-1 / p-\alpha}(1+\log n)^{\delta} .
$$

Proof of Theorem 2. The implication $\Rightarrow$ is covered by Theorem 1. For the converse implication $\Leftarrow$ we proceed similarly to the lower estimate in the proof of Theorem 1. We now have

$$
\begin{aligned}
& n^{1 / q-1 / p-\alpha}(1+\log n)^{\delta} \succeq e_{n}\left(D_{\sigma}\right) \geq e_{n}\left(R_{n}\right) \\
& \geq \sigma_{n} \cdot e_{n}\left(i d: l_{p}^{n} \rightarrow l_{q}^{n}\right) \sim \sigma_{n} \cdot n^{1 / q-1 / p},
\end{aligned}
$$

whence $\sigma_{n} \preceq n^{-\alpha}(1+\log n)^{\delta}$.

Before passing to the proof of Theorem 3 we give an auxiliary result.

Lemma 9. Let $w=\left(w_{n}\right)$ be a non-increasing weight sequence, let $1 \leq p<$ $q \leq \infty$ and set $\lambda:=1 / p-1 / q$. Then for all $n \in \mathbb{N}$

$$
e_{n}\left(D_{w^{\lambda}}: l_{p} \rightarrow l_{q}\right) \leq 4 e_{n}\left(D_{w}: l_{1} \rightarrow l_{\infty}\right)^{\lambda} .
$$

Proof. Let us start with the following simple but useful observation. It is obvious that the Banach spaces $l_{p}$ and $l_{p}(w), 1 \leq p \leq \infty, w=\left(w_{n}\right)$ an arbitrary weight sequence, are isometrically isomorphic, where the isomorphism is given by the diagonal operator $D_{w}, D_{w}\left(x_{n}\right)=\left(w_{n} x_{n}\right)$. For the entropy numbers this implies

$$
e_{n}\left(i d: l_{p} \rightarrow l_{q}(w)\right)=e_{n}\left(D_{w}: l_{p} \rightarrow l_{q}\right)=e_{n}\left(i d: l_{p}\left(w^{-1}\right) \rightarrow l_{q}\right),
$$

which means the weights can be shifted from the spaces to the operators.

Let us assume for the moment that $1<p<q<\infty$.

Step 1 . Setting $\theta:=1 / p$, we clearly have $\theta \in(0,1)$ and $\frac{1-\theta}{\infty}+\frac{\theta}{1}=\frac{1}{p}$ whence by Lemma 7

$$
\left[l_{\infty}, l_{1}\left(w^{-1}\right)\right]_{\theta}=l_{p}\left(w^{-\theta}\right) .
$$

Moreover, by shifting the weights Lemma 8 implies

$$
\begin{aligned}
& e_{n}\left(i d: l_{p} \rightarrow l_{\infty}\left(w^{\theta}\right)\right)=e_{n}\left(i d: l_{p}\left(w^{-\theta}\right) \rightarrow l_{\infty}\right) \\
& \leq 2\left\|i d: l_{\infty} \rightarrow l_{\infty}\right\|^{1-\theta} e_{n}\left(i d: l_{1}\left(w^{-1}\right) \rightarrow l_{\infty}\right)^{\theta} \\
& =2 e_{n}\left(D_{w}: l_{1} \rightarrow l_{\infty}\right)^{\theta} .
\end{aligned}
$$

Step 2. Next we set $\eta:=p / q$. Then $\eta \in(0,1)$ and $\frac{1-\eta}{\infty}+\frac{\eta}{p}=\frac{1}{q}$ whence Lemma 7 now gives

$$
\left[l_{\infty}\left(w^{\theta}\right), l_{p}\right]_{\eta}=l_{q}\left(w^{\theta(1-\eta)}\right)=l_{q}\left(w^{\lambda}\right) .
$$


Therefore Lemma 8 and Step 1 yield

$$
\begin{aligned}
& e_{n}\left(D_{w^{\lambda}}: l_{p} \rightarrow l_{q}\right)=e_{n}\left(i d: l_{p} \rightarrow l_{q}\left(w^{\lambda}\right)\right) \\
& \leq 2 e_{n}\left(i d: l_{p} \rightarrow l_{\infty}\left(w^{\theta}\right)\right)^{1-\eta}\left\|i d: l_{p} \rightarrow l_{p}\right\|^{\eta} \\
& =2 e_{n}\left(i d: l_{p} \rightarrow l_{\infty}\left(w^{\theta}\right)\right)^{1-\eta} \leq 4 e_{n}\left(D_{w}: l_{1} \rightarrow l_{\infty}\right)^{\lambda} .
\end{aligned}
$$

If $p=1$ (resp. $q=\infty$ ), then the first (resp. second) step can be omitted, and so we have the desired result in all cases.

Proof of Theorem 3. We adopt the notation $L_{\delta}$ for the diagonal operator $D_{\sigma}$ generated by the logarithmic sequence $\sigma_{n}=(1+\log n)^{-\delta}$ with $\delta>0$, i.e.,

$$
L_{\delta}\left(x_{n}\right)=\left((1+\log n)^{-\delta} x_{n}\right) .
$$

Upper estimate: We divide the proof into several steps.

Step 1 . First we consider the special case $p=1, q=\infty, \delta=1$. By Lemma 6 we have

$$
e_{n}\left(L_{1 / 2}: l_{1} \rightarrow l_{2}\right) \preceq n^{-1 / 2},
$$

and by Tomczak-Jaegermann's results [24] on the duality of entropy numbers this implies

$$
e_{n}\left(L_{1 / 2}: l_{2} \rightarrow l_{\infty}\right) \preceq n^{-1 / 2}
$$

therefore the multiplicativity of entropy numbers yields

$$
e_{2 n}\left(L_{1}: l_{1} \rightarrow l_{\infty}\right) \leq e_{n}\left(L_{1 / 2}: l_{1} \rightarrow l_{2}\right) \cdot e_{n}\left(L_{1 / 2}: l_{2} \rightarrow l_{\infty}\right) \preceq n^{-1},
$$

which is the desired upper estimate in our special case.

Step 2. Next we consider the case $p=1, q=\infty, \delta>1$. Here we write $\delta=\alpha+1$; clearly, $\alpha>0$. By the result of Gordon, König and Schütt [11], see our Remark 6, we have

$$
e_{n}\left(L_{\alpha}: l_{1} \rightarrow l_{1}\right) \sim(1+\log n)^{-\alpha},
$$

which leads, together with Step 1 and the multiplicativity of the entropy numbers, to the estimate

$$
e_{2 n}\left(L_{\delta}: l_{1} \rightarrow l_{\infty}\right) \leq e_{n}\left(L_{\alpha}: l_{1} \rightarrow l_{1}\right) e_{n}\left(L_{1}: l_{1} \rightarrow l_{\infty}\right) \preceq n^{-1} \cdot(1+\log n)^{-\alpha} .
$$

This is the assertion since $-\alpha=1 / p-1 / q-\delta$ in the present case.

Step 3 . In the case $p=1, q=\infty, 0<\delta<1$ we use the interpolation formula

$$
\left[l_{1}, l_{1}\left(w^{-1}\right)\right]_{\delta}=l_{1}\left(w^{-\delta}\right)
$$

with the special weight sequence $w=\left(w_{n}\right), w_{n}=1+\log n$. From the result in Step 1 for $\delta=1$ we conclude analogously to the proof of Lemma 9 that

$$
\begin{aligned}
& e_{n}\left(L_{\delta}: l_{1} \rightarrow l_{\infty}\right)=e_{n}\left(i d: l_{1}\left(w^{-\delta}\right) \rightarrow l_{\infty}\right) \\
& \leq 2\left\|i d: l_{1} \rightarrow l_{\infty}\right\|^{1-\delta} e_{n}\left(i d: l_{1}\left(\left(w^{-1}\right) \rightarrow l_{\infty}\right)^{\delta}\right. \\
& =2 e_{n}\left(L_{1}: l_{1} \rightarrow l_{\infty}\right)^{\delta} \preceq n^{-\delta} .
\end{aligned}
$$


Step 4. Finally, in the general case $1 \leq p<q \leq \infty, \delta>0$ the assertion follows from Interpolation Lemma 9 and the results in Steps 1-3.

Lower estimate: For arbitrary $n \in \mathbb{N}$, let $R_{n}$ denote the restriction of $D_{\sigma}$ to the coordinates $1, \ldots, n^{2}$. Then relation (1) and the multiplicativity of entropy numbers imply

$$
\begin{aligned}
& e_{n}\left(D_{\sigma}\right) \geq e_{n}\left(R_{n}\right) \geq \sigma_{n^{2}} \cdot e_{n}\left(i d: l_{p}^{n^{2}} \rightarrow l_{q}^{n^{2}}\right) \\
& \sim(1+\log n)^{-\delta} \cdot\left(\frac{1+\log n}{n}\right)^{1 / p-1 / q},
\end{aligned}
$$

and this shows the lower estimate for $\delta \geq \delta^{*}$. In the case $0<\delta<\delta^{*}$ we consider the restriction of $D_{\sigma}$ to the coordinates $1, \ldots, 2^{n}$. Then $\sigma_{2^{n}} \sim n^{-\delta}$ and, similarly to the above we obtain the desired lower estimate

$$
e_{n}\left(D_{\sigma}\right) \geq \sigma_{2^{n}} \cdot e_{n}\left(i d: l_{p}^{2^{n}} \rightarrow l_{q}^{2^{n}}\right) \sim n^{-\delta}
$$

Proof of Theorem 4. Theorem 4 can be derived from Theorem 3 in the same way as Theorem 2 from Theorem 1 and therefore the details are omitted.

\section{ACKNOWLEDGEMENT}

The author greatfully acknowledges the hospitality of Florida Atlantic University, Boca Raton, where the first draft of this paper was written.

\section{REFERENCES}

1. J. Bergh and J. LÖFström, Interpolation spaces. Springer-Verlag, Berlin, Heidelberg, New York, 1976.

2. B. CARL, Entropy numbers of diagonal operators with an application to eigenvalue problems. J. Approx. Theory 32(1981), 135-150.

3. B. CARL, I. Kyrezi, and A. PAJor, Metric entropy of convex hulls in Banach spaces. J. London Math. Soc. (2) 60(1999), 871-896.

4. B. Carl and I. Stephani, Entropy, compactness and approximation of operators. Cambridge Univ. Press, Cambridge, 1990.

5. F. Cobos and T. KüHN, Entropy numbers of embeddings of Besov spaces in spaces of Lipschitz type. J. Approx. Theory (to appear).

6. J. Creutzig and I. Steinwart, Metric entropy of convex hulls in type $p$ spaces - the critical case. Proc. Amer. Math. Soc. (to appear).

7. D. E. Edmunds and D. Haroske, Spaces of Lipschitz type, embeddings and entropy numbers. Dissertationes Math. 380(1999), 1-43.

8. D. E. Edmunds and D. D. Haroske, Embeddings in spaces of Lipschitz type, entropy and approximation numbers, and applications. J. Approx. Theory 104(2000), $226-271$.

9. D. E. Edmunds and H. Triebel, Function spaces, entropy numbers and differential operators. Cambridge Univ. Press, Cambridge, 1996.

10. F. GaO, Metric entropy of convex hulls. Israel J. Math. (2001) (to appear).

11. Y. Gordon, H. KÖNIG, and C. SchüTt, Geometric and probabilistic estimates for entropy and approximation numbers of operators. J. Approx. Theory 49(1987), 219239. 
12. H. KÖNIG, Eigenvalue distribution of compact operators. Birkhäuser, Basel, 1986.

13. T. KüHN, $\gamma$-Radonifying operators and entropy ideals. Math. Nachr. 107(1982), $53-58$.

14. T. KüHn, A lower estimate for entropy numbers. J. Approx. Theory 110(2001), 120124.

15. T. KüHN and T. SChOnBeK, Entropy numbers of diagonal operators between vectorvalued sequence spaces. J. London Math. Soc. (to appear).

16. M. Ledoux and M. Talagrand, Probability in Banach spaces. Springer-Verlag, Berlin, Heidelberg, New York, 1991.

17. H.-G. Leopold, Embeddings and entropy numbers in Besov spaces of generalized smoothness. Function Spaces (Poznan, 1998), Lecture Notes in Pure Appl. Math. 213, 323-336, Marcell Dekker, New York, 2000.

18. H.-G. LEOPOLD, Embeddings for general weighted sequence spaces and entropy numbers. Function Spaces, Differential Operators and Nonlinear Analysis, Proceedings of Conference held in Syöte, 1999, 170-186, Math. Inst. Acad. Sci. Czech Republic, Prague, 2000.

19. W. Linde and A. Pietsch, Mappings of Gaussian measures of cylindrical sets in Banach spaces. (Russian) Teor. Verojatnost. i Primenen. 19(1974), 427-487; English translation: Theory Probab. Appl. 19(1974), 445-460.

20. G. PIsIER, The volume of convex bodies and Banach space geometry. Cambridge Univ. Press, Cambridge, 1989.

21. A. Pietsch, Operator ideals. VEB Deutscher Verlag der Wiss. Berlin, 1978, and North-Holland, Amsterdam-New York, 1980.

22. C. SснÜтT, Entropy numbers of diagonal operators between symmetric Banach spaces. J. Approx. Theory 40(1984), 121-128.

23. V. N. Sudakov, Gaussian random processes, and measures of solid angles in Hilbert space. (Russian) Dokl. Akad. Nauk SSSR 197 (1971), 43-45; English transl.: Soviet Math. Dokl. 12(1971), 412-415.

24. N. TomczaK-Jaegermann, Dualité des nombres d' entropie pour des opérateurs à valeurs dans un espace de Hilbert. C. R. Acad. Sci. Paris, Ser. I 305(1987), 299-301.

25. H. Triebel, Fractals and spectra. Birkhäuser, Basel, 1997.

26. H. TRIEBEL, Interpolation theory, function spaces, differential operators. NorthHolland, Amsterdam, New York, Oxford, 1978.

(Received 15.07.2000; revised 22.03.2001)

Author's address:

Mathematisches Institut

Universität Leipzig

Augustusplatz 10/11, Leipzig

Germany

E-mail: kuehn@mathematik.uni-leipzig.de 